\begin{tabular}{|c|l|}
\hline Title & $\begin{array}{l}\text { On lower semicontinuity of a defect energy obtained by a singular limit of the Ginzburg-Landau type energy for } \\
\text { gradient fields }\end{array}$ \\
\hline Author(s) & A viles, P.; Giga, Y. \\
\hline Citation & Hokkaido University Preprint Series in Mathematics, 386, 1-21 \\
\hline Issue Date & 1997-7-1 \\
\hline DOI & 10.14943/83532 \\
\hline Doc URL & http:/hdl.handle.net/2115/69136 \\
\hline Type & bulletin (article) \\
\hline File Information & pre386.pdf \\
\hline
\end{tabular}

Instructions for use 
On lower semicontinuity of a defect energy obtained by a singular limit of the Ginzburg-Landau type energy for gradient fields

P. Aviles and Y. Giga

Series \#386. July 1997 


\section{HOKKAIDO UNIVERSITY \\ PREPRINT SERIES IN MATHEMATICS}

\#360 I. Tsuda and A. Yamaguchi, Singular-continuous nowhere-differentiable attractors in neural systems, 40 pages. 1996.

\#361 M. Nakamura and T. Ozawa, Low energy scattering for nonlinear Schrödinger equations in fractional order Sobolev spaces, 17 pages. 1996.

\#362 I. Nakamura, Hilbert schemes and simple singularities $E_{6}, E_{7}$ and $E_{8}, 21$ pages. 1996.

\#363 T. Mikami, Equivalent conditions on the central limit theorem for a sequence of probability measures on $R, 7$ pages. 1996.

\#364 S. Izumiya and T. Sano, Generic affine differential geometry of space curves, 23 pages. 1996.

\#365 T. Tsukada, Stability of reticular optical caustics, 12 pages. 1996.

\#366 A. Arai and M. Hirokawa, On the existence and uniqueness of ground states of a generalized spin-boson model, 40 pages. 1996.

\#367 A. Arai, A class of representations of the *-algebra of the canonical commutation relations over a Hilbert space and instability of embedded eigenvalues in quantum field models, 12 pages. 1996.

\#368 K. Ito, BV-solutions of a hyperbolic-elliptic system for a radiating gas, 33 pages. 1997.

\#369 M. Nakamura and T. Ozawa, Nonlinear Schrödinger equations in the Sobolev space of critical order, 20 pages. 1997 .

\#370 N.H. Bingham and A. Inoue, An Abel-Tauber theorem for Hankel transforms, 8 pages. 1997.

\#371 T. Nakazi and H. Sawada, The commutator ideal in Toeplitz algebras for uniform algebras and the analytic structure, 9 pages. 1997 .

\#372 M.-H. Giga and Y. Giga, Stability for evolving graphs by nonlocal weighted curvature, 70 pages. 1997.

\#373 T. Nakazi, Brown-Halmos type theorems of weighted Toeplitz operators, 14 pages. 1997.

\#374 J. Inoue and S.-E. Takahashi, On characterizations of the image of Gelfand transform of commutative Banach algebras, 30 pages. 1997.

\#375 L. Solomon and H. Terao, The double coxeter arrangement, 21 pages. 1997.

\#376 G. Ishikawa and T. Morimoto, Solution surfaces of Monge-Ampère equations, 15 pages. 1997.

\#377 G. Ishikawa, A relative transversality theorem and its applications, 16 pages. 1997.

\#378 J. Inoue and T. Nakazi, On the zeroes of solutions of an extremal problem in $H^{1}, 14$ pages. 1997.

\#379 Y. Giga and K. Ito, On pinching of curves moved by surface diffusion, 12 pages. 1997.

\#380 F. Hiroshima, Weak coupling limit removing an ultraviolet cut-off for a Hamiltonian of particles interacting with a scalar field, 39 pages. 1997.

\#381 Y. Giga, S. Matsui and Y. Shimizu, On estimates in Hardy spaces for the Stokes flow in a half space, 13 pages. 1997.

\#382 A. Arai, A new estimate for the ground state energy of Schrödinger operators, 12 pages. 1997.

\#383 M. Nakamura and T. Ozawa, The Cauchy problem for nonlinear wave equations in the Sobolev space of critical order, 24 pages. 1997.

\#384 K. Ito, Asymptotic stability of planar rarefaction wave for scalar viscous conservation law, 8 pages. 1997.

\#385 A. Arai, Representation-theoretic aspects of two-dimensional quantum systems in singular vector potentials: canonical commutation relations, quantum algebras, and reduction to lattice quantum systems, 32 pages. 1997. 


\title{
On lower semicontinuity of a defect energy obtained by a singular limit of the Ginzburg-Landau type energy for gradient fields
}

\author{
Patricio Aviles* and Yoshikazu Giga \\ Department of Mathematics \\ Hokkaido Univiversity \\ Sapporo 060, Japan
}

\section{Introduction}

We are concerned with a defect energy measuring the strength of jump discontinuities of gradient vector fields. A typical form of a defect energy for a gradient vector field $\nabla u$ in a bounded domain $\Omega$ in $\mathbf{R}^{n}$ is

$$
J^{\beta}(\nabla u)=\int_{\Sigma}|[\nabla u]|^{\beta} d \mathcal{H}^{n-1}
$$

with $\beta>0$, where $\Sigma$ denotes the jump discontinuity of $\nabla u$ and $[\nabla u]$ denotes the difference of $\nabla u$ of each side of $\Sigma ; \mathcal{H}^{n-1}$ denotes the $n-1$ dimensional Hausdorff measure. There may be many Lipschitz solutions of the eikonal equation

$$
|\nabla u|=1 \text { in } \Omega \text { with } u=0 \text { on } \partial \Omega \text {. }
$$

There are several selection principles of solutions depending on physical situation. A widely known selection principle is provided by the theory of viscosity solutions which selects the distance function from the boundary as a unique viscosity solution of (1.2) [L]. However, there may be another way to select solutions by minimising a defect energy $J^{\beta}$, as arised in the modelling of smectic liquid crystals [AG1], [SK] or of the blistering of thin films [OG] or of the magnetic walls in soft magnetic thin films $[\mathrm{VB}],\left[\mathrm{VB}^{2}\right]$. As already shown in [AG5] the viscosity solution of (1.2) may not minimise $J^{\beta}(\beta>0)$ among all solutions of (1.2) for some two-dimensional bounded non-convex domain $\Omega$ with Lipschitz boundary $\partial \Omega$. However, for a convex polygonal domain $\Omega$ it is shown in [AG5] that the viscosity solution of (1.2) minimises $J^{1}$

\footnotetext{
* Fermanent address, University of Ilinois at Urbana-Champaign, Illinois 61801 , USA
} 
among all gradient vector fields with bounded variation satisfying (1.2). The proof is based on rank one properties of the Hessian $\nabla \nabla u$ [Al], [AG2]. The problem for general convex domain as well as for general energy $J^{\beta}$ seems to be left open although there are several interesting observation by W. Jin and R. Kohn [JK].

The existence of minimisers of $J^{\beta}$ subject to (1.2) is not known in general. To attack this problem by the direct method the crucial step is to show

(i) lower semicontinuity of $J^{\beta}$;

(ii) compactness of a set of $\nabla u$ on which $J^{\beta}(\nabla u)$ is bounded;

under the same topology of gradient vector fields satisfying (1.2). If $n=1, J^{\beta}$ equals $2^{\beta}$ times the total variation of $u_{x}=\nabla u$ with $\left|u_{x}\right|=1$. Thus it is easy to show (i) and (ii) under $L^{1}$ convergence of $u_{x}$ by the general theory of functions of bounded variation [Giu]. However, both problems are nontrivial when $n \geq 2$. In this paper we focus the problem of lower semicontinuity of $J^{\beta}$ under $L^{1}$ convergence of unit length gradient vector fields.

The main goal of this paper is to prove that $J^{3}$ is lower semicontinuous under the $L^{1}$ convergence of $\nabla u$ provided that $u$ solves $|\nabla u|=1$ in $\Omega$ and that $\nabla u$ has bounded variation (shortly, $u \in B G V(\Omega)$ ) when $n=2$. We do not need to assume that $u=0$ on $\partial \Omega$. The key step is to establish a representation formula of $J^{3}(\nabla u)$ when $|\nabla u|=1$ in $\Omega$ (Theorem 2.1). Loosely speaking, the formula reads:

$$
J^{3}(\nabla u)=4 \sup \sum_{i=1}^{m} \int_{\Omega_{i}}\left|\partial_{\xi}\left(u_{\xi}^{3}\right)-\partial_{\eta}\left(u_{\eta}^{3}\right)\right|
$$

where sup is taken over all finite Borel decomposition $\left\{\Omega_{i}\right\}_{i=1}^{m}$ and orthogonal basis $\{\xi, \eta\}$ obtained by a rotation of the standard basis $\left\{e_{1}, e_{2}\right\}$ of $\mathbf{R}^{2} ; u_{\xi}=\partial_{\xi} u$ denotes the directional derivative and $|\mu|$ denotes the total variation measure of a measure $\mu$. A crucial step to obtain (1.3) is an observation that

$$
\begin{aligned}
& \partial_{\xi}\left(u_{\xi}^{3}\right)-\partial_{\eta}\left(u_{\eta}^{3}\right) \\
= & \frac{3}{2}\left(1-u_{\xi}^{2}-u_{\eta}^{2}\right)\left(u_{\xi \xi}-u_{\eta \eta}\right)=0
\end{aligned}
$$

outside $\Sigma$ if $|\nabla u|=1$ in $\Omega$. The first identity is essentially contained in [JK].

Our proof also applies to get lower semicontinuity of modified energy:

$$
J_{+}^{\beta}(\nabla u)=\int_{\Sigma}|[\nabla u]|^{\beta} \cdot \alpha(\nabla u) d \mathcal{H}^{1}
$$


provided that $\beta=3$; here $\alpha=1$ if $\nabla u$ in near $\Sigma$ in one side of $\Sigma$ points out the same side of $\Sigma$ and otherwise $\alpha=0$. What is surprising us is that $J_{+}^{\beta}$ for $\beta>3$ is not lower semicontinuous under the $L^{1}$-convergence of $\nabla u$ with $|\nabla u|=1$ in $\Omega$ and with bounded total variation. We shall give an explicit counterexample in Remark 2.8. From this example, it is natural to conjecture that $J^{\beta}$ for $\beta>3$ is not lower semicontinuous. So our proof should not be extended to the case $\beta>3$. Similar counterexample is pointed out independently and concurrently by W. Jin and R. Kohn.

In this paper we do not investigate questions related to (ii). We do not even know whether or not the set $Y$ of $\nabla u$ with $u \in B G V(\Omega)$ solving $|\nabla u|=1$ in $\Omega$ with $\sup _{Y} J^{3}<\infty$ is always closed in $L^{1}$.

Our representation formula is also important to study Gamma limit (see e.g. $[\mathrm{DM}]$ ) of the singularity perturbed variational problem with the Ginzburg-Landau type energy:

$$
E_{\varepsilon}(\nabla u)=\frac{1}{2} \int_{\Omega} \varepsilon^{-1} W(\nabla u)+\varepsilon|\nabla \nabla u|^{2}, W(p)=\left.|| p\right|^{2}-\left.1\right|^{\rho}
$$

in a two dimensional bounded domain $\Omega$ with $u=0$ on $\partial \Omega$ when $\rho=2$. Since the Euler-Lagrange equation is fourth order we are entitled to impose another boundary condition. The natural choice seems to be $\partial u / \partial \mathrm{n}=-1$, where $\mathrm{n}$ is the unit outward normal of $\partial \Omega$. We are interested in the behaviour of $E_{\varepsilon}$ as $\varepsilon \rightarrow 0$. If we admit the one-dimensional ansatz saying that $\nabla u$ is almost parallel near internal transition layer, formal analysis for $\rho=2$ done in [AG1], [OG] suggests that the problem has Gamma limit

$$
\begin{gathered}
\bar{J}=\int_{\Sigma} \int_{-b}^{b}\left|1-\left(a^{2}+\tau^{2}\right)\right|^{\rho / 2} d \tau d \mathcal{H}^{1} \\
\alpha=\left|(\nabla u)_{\tan }\right|, b=\left(1-a^{2}\right)^{1 / 2}
\end{gathered}
$$

where $(\nabla u)_{\tan }$ denotes the tangential component of $\nabla u$ to $\Sigma$. Since $|\nabla u|=1$, we see

$$
\vec{J}=c J^{\rho+1} \text { with } c=\frac{1}{2^{\rho+1}} \int_{-1}^{1}\left(1-s^{2}\right)^{\rho / 2} d s .
$$

The value $c=1 / 6$ when $\rho=2$. Although it was conjectured a long time ago in [AG1] that the Gamma limit of $E_{\varepsilon}$ with $\rho=2$ equals $\vec{J}=J^{3} / 6$, it has been left 
open. Using our representation formula (1.3), we prove that

$$
J^{3}(\nabla u) / 6 \leq \liminf _{\varepsilon \rightarrow 0} E_{\varepsilon}\left(\nabla u^{\varepsilon}\right)
$$

for $\rho=2$ provided that $\nabla u$ fulfills (1.2) with bounded variation of $\nabla u$ and that $\nabla u^{\varepsilon}$ converges to $\nabla u$ in $L^{p}(\Omega)$ for $p \geq 3$. This provides an estimate from below of the Gamma limit of $E_{\varepsilon}$ at least $L^{3}(\Omega)$ sense. Although we do not study in this paper, in many examples $\nabla u$ has an approximate sequence $\nabla u^{\varepsilon}$ satisfying the boundary condition for $E_{\varepsilon}$ with

$$
\lim _{\varepsilon \rightarrow 0} E_{\varepsilon}\left(\nabla u^{\varepsilon}\right)=J^{3}(\nabla u) / 6
$$

if $\nabla u$ is sufficiently regular away from $\Sigma$. Thus our estimate (1.5) is sharp.

Since we believe that $J^{\beta}$ for $\beta>3$ is not lower semicontinuous, for $\rho>2$ the Gamma limit of $E_{\varepsilon}$ must be different from $\bar{J}$. This suggests that the behaviour of $E_{\varepsilon}$ as $\varepsilon \rightarrow 0$ for $\rho=2$ is rather special and there arises no microstructure which may break one dimensional ansatz. For $\rho=2$ we also prove that the Gamma limit $E_{\varepsilon}$ is the same as that of

$$
\hat{E_{\varepsilon}}(\nabla u)=\frac{1}{2} \int_{\Omega} \varepsilon^{-1} W(\nabla u)+\varepsilon|\Delta u|^{2}
$$

by extending an integral identity obtained in [JK].

Convergence problem of $E_{\varepsilon}$ for gradient vector fields is not well studied compared with scalar field. When $W$ has isolated equal minima, the problem was studied by $[\mathrm{KM} 1,2]$ where it was shown that the microstructure arises. For our $E_{\varepsilon}$ with $\rho=2$, W. Jin and R. Kohn [JK] calculated the limit of minimal value of $E_{\varepsilon}$ and proved that results are compatible with one-dimensional ansatz for several examples of domain. However, if there is anisotropy in $|\nabla u|$ for example $|\nabla u|^{2}$ is replaced by

$$
u_{x}^{2}+\lambda u_{y}^{2} \text { for small } \lambda>0
$$

they showed that the one-dimensional ansatz is not longer valid.

\section{Representation and lower semicontinuity of defect energy}

Let $\Omega$ be a bounded domain in $\mathbf{R}^{2}$. Let $L^{1}(\Omega)$ denote the space of integrable functions on $\Omega$. We do not distinguish spaces of vector-valued functions from that 
of scalar-valued functions. Let $B V(\Omega)$ denote the space of functions with bounded variation, i.e.,

$$
B V(\Omega)=\left\{w \in L^{1}(\Omega)\right.
$$

each component of $\nabla w$ is a finite (signed) Radon measure on $\Omega$ \},

where $\nabla w$ denotes the distributional gradient of $w$. Let $B G V(\Omega)$ be the space of real-valued functions whose gradient belongs to $B V(\Omega)$, i.e.,

$$
B G V(\Omega)=\left\{u \in L^{1}(\Omega) ; \nabla u \in B V(\Omega)\right\}
$$

For $u \in B G V(\Omega)$ it is well-known (see [F], [Giu], [S]) that the Hessian $\nabla \nabla u$ is a $2 \times 2$ matrix of Radon measures decomposed as

$$
\begin{aligned}
\nabla \nabla u= & \nabla \nabla u\left\lfloor\Omega_{0}+\nabla \nabla u\left\lfloor\left(\Omega \backslash\left(\Omega_{0} \cup \Sigma\right)\right)\right.\right. \\
& +\nu \otimes\left(\nabla u^{+}-\nabla u^{-}\right) \mathcal{H}^{1}\lfloor\Sigma
\end{aligned}
$$

where $\otimes$ denotes the tensor product of vectors. Here $\Sigma$ denotes the set of jump discontinuity of $\nabla u$ and $\nu$ represents a $\mathcal{H}^{1}\lfloor\Sigma$ measurable unit normal vector field on $\Sigma$. The vectors $\nabla u^{ \pm}$are the trace of $u$ on $\Sigma$ defined by

$$
\nabla u^{ \pm}(x)=\lim _{\varepsilon \downarrow 0} \nabla u(x \pm \varepsilon \nu(x))
$$

and $\mathcal{H}^{1}$ denotes the one dimensional Hausdorff measure. By $\mu\lfloor A$ we mean a measure on $\Omega$ defined by $(\mu\lfloor A)(D)=\mu(A \cap D)$ for $D \subset \Omega$, where $\mu$ is a measure. The part $\nabla \nabla u\left\lfloor\Omega_{0}\right.$ is absolutely continuous with respect to the Lebesgue measure $\mathcal{L}^{2}$ and $\mathcal{L}^{2}\left(\Omega_{0}\right)=\mathcal{L}^{2}(\Omega)$. The last two terms in (2.1) are singular with respect to $\mathcal{L}^{2}$.

In [AG5] several integrals measuring the size of jump discontinuities of $\nabla u$ are studied for $u \in B G V(\Omega)$. We recall one of them. Let $J^{\beta}(\beta>0)$ be of form

$$
J^{\beta}=\int_{\Sigma}|[\nabla u]|^{\beta} d \mathcal{H}^{1},
$$

where $[\nabla u]=\nabla u^{+}-\nabla u^{-}$. This quantity only depends on $u$ through its gradient so we often write it by $J^{\beta}(\nabla u)$. If $|\nabla u|$ is bounded on $\Omega, J^{\beta}$ for $\beta \geq 1$ is always finite for $u \in B G V(\Omega)$, since $J^{\beta}$ is dominated by a constant multiple of $J^{1}$ which is dominated by the total variation of $\nabla \nabla u$. 
The goal of this section is to give representation of $J^{3}$ and prove the lower semicontinuity of $J^{3}$ under the $L^{1}$-convergence of gradients of $u \in B G V(\Omega)$ when $\nabla u$ solves the eikonal equation $|\nabla u|=1$ in $\Omega$. For this purpose we use a vector field associated to $\nabla u$ which turns to be the same as introduced by W. Jin and $R$. Kohn $[\mathrm{JK}]$ when $|\nabla u|=1$ in $\Omega$. We set

$$
\Xi(p, q)=\left(\frac{2}{3} p^{3},-\frac{2}{3} q^{3}\right)
$$

and define

$$
G_{I}(\nabla u)=\operatorname{div}\left(\Xi\left(u_{x}, u_{y}\right)\right), \nabla u=\left(u_{x}, u_{y}\right) .
$$

Since $\Xi$ is $C^{1}$, the chain rule by A. I. Volpert [V] implies that $G_{I}(\nabla u)$ is a signed Radon measure on $\Omega$ for $u \in B G V(\Omega)$ if $|\nabla u|$ is bounded on $\Omega$. We need to rotate this measure. Let $B=\{\xi, \eta\}$ denote an orthonormal basis of $\mathbf{R}^{\mathbf{2}}$ with the same orientation as $I=\left\{e_{1}, e_{2}\right\}$, where $e_{1}=(1,0), e_{2}=(0,1)$, i.e., there is a rotation matrix $U$ such that $U e_{1}=\xi, U e_{2}=\eta$. Let $\mathcal{B}_{+}$denote the sets of all orthonormal basis with the same orientation as $I$. For $B \in \mathcal{B}_{+}$we define

$$
G_{B}(\nabla u)=\operatorname{div}^{B}\left(\Xi\left(u_{\xi}, u_{\eta}\right)\right)=\partial_{\xi}\left(\frac{2}{3} u_{\xi}^{3}\right)-\partial_{\eta}\left(\frac{2}{3} u_{\eta}^{3}\right),
$$

where $u_{\xi}=\partial_{\xi} u, u_{\eta}=\partial_{\eta} u$ and $\partial_{\xi}, \partial_{\eta}$ denote the directional derivative; here $\operatorname{div}^{B}$ denotes the divergence in the coordinate system associated to $B$. We decompose the domain and on each decomposed pieces we consider this rotated measure $G_{B}$. To be precise let $\Lambda$ denote the totality of all finite Borel decomposition of $\Omega$, i.e., each element of $\Lambda$ consists of a finite set of disjoint Borel sets $\left\{\Omega_{i}\right\}_{i=1}^{m}$ in $\Omega$ with $\cup_{i=1}^{m} \Omega_{i}=\Omega$. Let $\Lambda_{0}$ be the set of $\left\{\Omega_{i}\right\}_{i=1}^{m} \in \Lambda$ with the property that each $\Omega_{i}$ is of form

$$
\Omega_{i}=\{(x, j) ; a \leq x<b, c \leq y<d\} \cap \Omega
$$

with some $a, b, c, d \in \mathbf{R}$. We are now in position to state our main results.

Theorem 2.1 (Representation). Assume that $u \in B G V(\Omega)$ satisfies $|\nabla u|=1$ $\left(\mathcal{L}^{2}-\right.$ a.e. $)$ in $\Omega$. Then

(i)

$$
\int_{\Sigma \cap A}|[\nabla u]|^{3} d \mathcal{H}^{1}=6 \sup \left\{\sum_{i=1}^{m} \int_{\Omega \cap A} d \mid G_{B_{i}}(\nabla u)\left\lfloor\Omega_{i} \mid ;\left\{\Omega_{i}\right\} \in \Lambda, B_{i} \in \mathcal{B}_{+}\right\}\right.
$$


for a set $A$ in $\Omega$, where $|\mu|$ denotes the total variation measure of a measure $\mu$. In particular

$$
J^{3}(\nabla u)=6 \sup \left\{\sum_{i=1}^{m} \int_{\Omega} d \mid G_{B_{i}}(\nabla u)\left\lfloor\Omega_{i} \mid ;\left\{\Omega_{i}\right\} \in \Lambda, B_{i} \in \mathcal{B}_{+}\right\} .\right.
$$

Both formulas are still valid even if $\Lambda$ is replaced by $\Lambda_{0}$.

(ii)

$$
\begin{gathered}
J^{3}(\nabla u)=6 \sup \left\{\sum_{i=1}^{m}\left|\int_{\Omega_{i}} \nabla_{B_{i}} \varphi_{i} \cdot \Xi\left(u_{\xi_{i}}, u_{\eta_{i}}\right) d \mathcal{L}^{2}\right|\right. \\
\left.\varphi_{i} \in \Phi_{i},\left\{\Omega_{i}\right\}_{i=1}^{m} \in \Lambda_{0} \text { and } B_{i} \in \mathcal{B}_{+}\right\},
\end{gathered}
$$

where $\Phi_{i}$ is the set of real-valued $C^{1}$ functions $\varphi$ compactly supported in int $\Omega_{i}$ (i.e. $\varphi \in C_{0}^{1}$ (int $\left.\Omega_{i}\right)$ ) that satisfies $|\varphi| \leq 1$ in $\Omega_{i} ; \nabla_{B_{i}}$ denotes the gradient in the coordinates associated to $B_{i}=\left\{\xi_{i} \eta_{i}\right\}$.

Theorem 2.2 (Lower semicontinuity). Assume that $u^{\varepsilon} \in B G V(\Omega)(\varepsilon \geq 0)$ satisfies $\left|\nabla u^{\varepsilon}\right|=1$ ( $\mathcal{L}^{2}$-a.e.) in $\Omega$. If $\nabla u^{\varepsilon}$ converges to $\nabla u^{0}$ in $L^{1}(\Omega)$ (or $\mathcal{L}^{2}$-a.e.), then

$$
J^{3}\left(\nabla u^{0}\right) \leq \liminf _{\varepsilon \rightarrow 0} J^{3}\left(\nabla u^{\varepsilon}\right) .
$$

Proof that Theorem 2.1 implies Theorem 2.2. Suppose that $\nabla u^{\varepsilon} \rightarrow \nabla u^{0}$ in $L^{1}(\Omega)$ as $\varepsilon \rightarrow 0$. We may assume that $\nabla u^{\varepsilon} \rightarrow \nabla u^{0}$ in $\Omega\left(\mathcal{L}^{2}\right.$-a.e.) by taking a subsequence. Since $\left|\nabla u^{\varepsilon}\right|$ is bounded, the dominated convergence theorem implies that

$$
\int_{\Omega_{i}} \nabla_{B_{i}} \varphi_{i} \cdot \Xi\left(u_{\xi_{i}}^{0} u_{\eta_{i}}^{0}\right) d \mathcal{L}^{2}=\lim _{\varepsilon \downarrow 0} \int_{\Omega_{i}} \nabla_{B_{i}} \varphi_{i} \cdot \Xi\left(u_{\xi_{i}}^{\varepsilon} u_{\eta_{i}}^{\varepsilon}\right) d \mathcal{L}^{2} .
$$

Since the supremum operation is lower semicontinuous under convergence, the representation (2.7) now yields (2.8).

To establish the representation foumulas (2.5), (2.6) we prepare several estimates. Lemma 2.3. $6\left|G_{B}(\nabla u)\right| \leq|[\nabla u]|^{3} \mathcal{H}^{1}\lfloor\Sigma$ as measures, where $u \in B G V(\Omega)$ with jump discontinuities $\Sigma$ fulfilles $|\nabla u|=1$ ( $\mathcal{L}^{2}$-a.e.) in $\Omega$ and $B \in \mathcal{B}_{+}$.

Proof. By rotation we may assume that $B=\left\{e_{1}, e_{2}\right\}=I$. Let $A$ be a set in $\Omega \backslash \Sigma$. Since $|\nabla u|=1$ on $\Omega$,

$$
\Xi\left(u_{x}, u_{y}\right)=\left(u_{x}\left(1-u_{y}^{2}-\frac{u_{x}^{2}}{3}\right),-u_{y}\left(1-u_{x}^{2}-\frac{u_{y}^{2}}{3}\right)\right)
$$


the right hand side is the vector field introduced by W. Jin and R. Kohn [JK]. As observed in [JK] at least formally a direct computation yields

$$
\operatorname{div}\left(\Xi\left(u_{x}, u_{y}\right)\right)=\left(1-u_{x}^{2}-u_{y}^{2}\right)\left(u_{x x}-u_{y y}\right) .
$$

By the chain role for functions with bounded variation (A. I. Volpert [V]) (2.10) implies that

$$
\operatorname{div}\left(\Xi\left(u_{x}, u_{y}\right)\right)\lfloor A=0
$$

since $|\nabla u|=1$ we may assume that $\nabla u$ is approximately continuous outside $\Sigma$ and $\Sigma \cap A=\emptyset$ so that on $A$ the right hand side of (2.10) equals zero. Thus

$$
\left|G_{I}(\nabla u)\right|\lfloor A
$$

is identically zero if $A \cap \Sigma=\emptyset$.

It remains to prove that

$$
6 \int_{\Sigma} d\left|G_{I}(\nabla u)\right| \leq \int_{\Sigma}|[\nabla u]|^{3} d \mathcal{H}^{1}
$$

Since

$$
\int_{\Sigma} d\left|G_{I}(\nabla u)\right|=\frac{2}{3} \int_{\Sigma}\left|\left(\left(u_{x}^{+}\right)^{3}-\left(u_{x}^{-}\right)^{3}\right) \nu_{x}-\left(\left(u_{y}^{+}\right)^{3}-\left(u_{y}^{-}\right)^{3}\right) \nu_{y}\right| d \mathcal{H}^{1},
$$

to show (2.11) it remains to prove that

$$
4\left|\left(\left(u_{x}^{+}\right)^{3}-\left(u_{x}^{-}\right)^{3}\right) \nu_{x}-\left(\left(u_{y}^{+}\right)^{3}-\left(u_{y}^{-}\right)^{3}\right) \nu_{y}\right| \leq\left(\left(u_{x}^{+}-u_{x}^{-}\right)^{2}+\left(u_{y}^{+}-u_{y}^{-}\right)^{2}\right)^{3 / 2}
$$

where $\nu=\left(\nu_{x}, \nu_{y}\right)$. We set

$$
\nu=(\cos \sigma, \sin \sigma), \nabla u^{+}=(\cos (\sigma+\theta), \sin (\sigma+\theta))
$$

so that

$$
\begin{aligned}
\nabla u^{-} & =(\cos (\sigma-\pi-\theta), \sin (\sigma-\pi-\theta)) \\
& =(-\cos (\sigma-\theta),-\sin (\sigma-\theta)) .
\end{aligned}
$$

By additive formulas (for trigonometric functions) we see that

$$
\begin{aligned}
\left(u_{x}^{+}\right)^{3} & =\cos ^{3}(\sigma+\theta)=\cos (\sigma+\theta)\left(1-\sin ^{2}(\sigma+\theta)\right) \\
& =\frac{1}{2} \cos (\sigma+\theta)(1+\cos 2(\sigma+\theta)) \\
\left(u_{x}^{-}\right)^{3} & =-\cos ^{3}(\sigma-\theta)=-\frac{1}{2} \cdot \cos (\sigma-\theta)(1+\cos 2(\sigma-\theta)) .
\end{aligned}
$$


Thus again using additive formulas we obtain

$$
\begin{aligned}
\left(u_{x}^{+}\right)^{3}-\left(u_{x}^{-}\right)^{3}= & \frac{1}{2}\{\cos (\sigma+\theta)+\cos (\sigma-\theta) \\
& +\cos (\sigma+\theta) \cos 2(\sigma+\theta)+\cos (\sigma-\theta) \cos 2(\sigma-\theta)\} \\
= & \frac{1}{2}\left\{2 \cos \sigma \cos \theta+\frac{1}{2}\{\cos (3(\sigma+\theta))+\cos (-(\sigma+\theta))\}\right. \\
& \left.+\frac{1}{2}\{\cos (3(\sigma-\theta))+\cos (-(\sigma-\theta))\}\right\} \\
= & \frac{1}{2}\{3 \cos \sigma \cos \theta+\cos 3 \sigma \cos 3 \theta\}
\end{aligned}
$$

Similar calculation deduces

$$
\left(u_{y}^{+}\right)^{3}-\left(u_{y}^{-}\right)^{3}=\frac{1}{2}\{3 \sin \sigma \cos \theta-\sin 3 \sigma \cos 3 \theta\}
$$

We now observe that

$$
\begin{aligned}
P= & \left(\left(u_{x}^{+}\right)^{3}-\left(u_{x}^{-}\right)^{3}\right) \nu_{x}-\left(\left(u_{y}^{+}\right)^{3}-\left(u_{y}^{-}\right)^{3}\right) \nu_{y} \\
= & \frac{1}{2}\{3 \cos \sigma \cos \sigma \cos \theta-3 \sin \sigma \sin \sigma \cos \theta \\
& +\cos 3 \sigma \cos 3 \theta \cos \sigma-(-\sin 3 \sigma \cos 3 \theta) \sin \sigma\} \\
= & \frac{1}{2}\{3 \cos \theta \cos 2 \sigma+\cos 3 \theta \cos (3 \sigma-\sigma)\} \\
= & \frac{1}{2} \cos 2 \sigma\{3 \cos \theta+\cos 3 \theta\}=\frac{4}{2} \cos 2 \sigma \cos ^{3} \theta .
\end{aligned}
$$

Since

$$
\begin{aligned}
\|\left.[\nabla u]\right|^{2} & =(\cos (\sigma+\theta)+\cos (\sigma-\theta))^{2}+(\sin (\sigma+\theta)+\sin (\sigma-\theta))^{2} \\
& =(2 \cos \sigma \cos \theta)^{2}+(2 \sin \sigma \cos \theta)^{2}=4 \cos ^{2} \theta
\end{aligned}
$$

our formula for $P$ implies

$$
4|P|=8|\cos 2 \sigma|\left|\cos ^{3} \theta\right| \leq 8\left|\cos ^{3} \theta\right|=|[\nabla u]|^{3}
$$

which is the same as (2.12). Thus the estimate in Lemma 2.3 has been proved.

The proof in particular yields: 
Lemma 2.4. For $u \in B G V(\Omega)$ satisfying $|\nabla u|=1\left(\mathcal{L}^{2}\right.$-a.e. $)$ in $\Omega$ the identity

$$
\begin{aligned}
\int_{\Sigma \cap A} d\left|G_{B}(\nabla u)\right| & =\frac{4}{3} \int_{\Sigma \cap A}|\cos 2 \sigma|\left|\cos ^{3} \theta\right| d \mathcal{H}^{1} \\
& =\frac{1}{6} \int_{\Sigma \cap A}|\cos 2 \sigma||[\nabla u]|^{3} d \mathcal{H}^{1}
\end{aligned}
$$

holds for a subset $A$ of $\Omega$ with $B=\{\xi, \eta\}$. Here $\sigma$ and $\sigma+\theta$ are arguments of $\nu$ and $\nabla_{B} u^{+}$with respect the coordinate system associated to $B$, respectively. (In the case $B=I, \sigma$ and $\theta$ are the same as given in (2.13).)

Proof of Theorem 2.1. $\quad$ (i) By Lemma 2.3 it suffices to prove that for each $\delta>0$ there is $\left\{\Omega_{i}\right\}_{i=1}^{m} \in \Lambda_{0}$ and $B_{i} \in \mathcal{B}_{+}$such that

$$
\int_{\Sigma \cap A}|[\nabla u]|^{3} d \mathcal{H}^{1} \leq \sum_{i=1}^{m} 6 \int_{\Sigma \cap A} d \mid G_{B_{i}}\left\lfloor\Omega_{i} \mid+\delta\right.
$$

for every subset $A$ in $\Omega$. We may assume that $\int_{\Sigma}|[\nabla u]|^{3} d \mathcal{H}^{1} \neq 0$. Since the jump discontinuity $\Sigma$ of $\nabla u$ is countably one rectifiable (see e.g. [DG1], [F], [Giu], [S], (AG2]), $\Sigma$ is of form

$$
\Sigma=\bigcup_{\ell=1}^{\infty} \Sigma_{\ell} \cup \Sigma_{0} \quad \text { (disjoint union) }
$$

with $\mathcal{H}^{1}\left(\Sigma_{0}\right)=0$; the set $\Sigma_{\ell}$ is compact and lies on some $C^{1}$ curve $C_{\ell}$ embedded in $\mathbf{R}^{2}$.

Since $|[\nabla u]|^{3}$ is bounded on $\Sigma$, for each $\delta>0$ there is $N>0$ (independent of $A$ ) that satisfies

$$
\left.\int_{\Sigma \cap A}|[\nabla u]|^{3}\left|d \mathcal{H}^{1} \leq \sum_{\ell=1}^{N} \int_{\Sigma_{\ell} \cap A}\right|[\nabla u]\right|^{3} d \mathcal{H}^{1}+\delta / 2
$$

Since $\Sigma_{\ell}$ is compact and lies on a $C^{1}$ hypersurface $C_{\ell}$, for each small $\omega$, say $0<$ $\omega<\pi / 4$, there is $\left\{\Omega_{i}\right\}_{i=1}^{m} \in \Lambda_{0}$ with the property that the set $\Theta_{\ell i}$ of arguments of $\nu$ in $\Omega_{i} \cap \Sigma_{\ell}(1 \leq \ell \leq N)$ contained in an interval of length less than $\omega$. On each $\Omega_{i}$ we take $B_{i}=\left\{\xi_{i}, \eta_{i}\right\} \in \mathcal{B}_{+}$such that the argument $\xi_{i} \in \Theta_{\ell i}$. By Lemma 2.4 and Lemma 2.3 we see that

$$
\begin{aligned}
& \int_{\Sigma_{\ell} \cap A \cap \Omega_{i}}|[\nabla u]|^{3}\left|d \mathcal{H}^{1} \leq \frac{6}{\cos 2 \omega} \int_{\Sigma_{\ell} \cap A} d\right| G_{B_{i}}(\nabla u)\left\lfloor\Omega_{i} \mid\right. \\
& \leq 6 \int_{\Sigma_{\ell} \cap A} d \mid G_{B_{i}}(\nabla u)\left\lfloor\Omega _ { i } | + ( \frac { 1 } { \operatorname { c o s } 2 \omega } - 1 ) \int _ { \Sigma } | [ \nabla u ] | ^ { 3 } d \left(\mathcal{H}^{1}\left\lfloor\Omega_{i}\right) .\right.\right.
\end{aligned}
$$


By (2.15) this yields

$$
\begin{aligned}
& \left.\int_{\Sigma \cap A}|| \nabla u\right|^{3}\left|d \mathcal{H}^{1} \leq 6 \sum_{i=1}^{m} \int_{\Sigma \cap A} d\right| G_{B_{i}}(\nabla u)\left\lfloor\Omega_{i} \mid+\delta / 2\right. \\
& +N\left(\frac{1}{\cos 2 \omega}-1\right) \int_{\Sigma}|[\nabla u]|^{3} d \mathcal{H}^{1} .
\end{aligned}
$$

We take $\omega$ small so that the last term is dominated by $\delta / 2$. Thus we obtain (2.14).

(ii) We may take $\Lambda=\Lambda_{0}$ in (2.6). Since $J^{3}(\nabla u)$ is finite, we may assume that $G_{B_{i}}\left\lfloor\partial \Omega_{i}=0\right.$ in $\Omega$ in (2.6) by shifting edges of rectangles. Since int $\Omega_{i}$ is not empty if $\left\{\Omega_{i}\right\}_{i=1}^{m} \in \Lambda_{0}$, for $u \in B G V(\Omega)$ the Riesz representation together with integration by parts yields

$$
\begin{aligned}
\int_{\Omega_{i}} d\left|G_{B_{i}}(\nabla u)\right| & =\sup \left\{\left|\int_{\Omega_{i}} \varphi d\right| G_{B_{i}}(\nabla u) \mid ; \varphi \in \Phi\right\} \\
& \left.=\sup \left|\int_{\Omega_{i}} \nabla_{B_{i}} \varphi \cdot \Xi\left(u_{\xi_{i}}, u_{\eta_{i}}\right) d \mathcal{L}^{2}\right| ; \varphi \in \Phi_{i}\right\},
\end{aligned}
$$

provided that $G_{B_{i}}\left\lfloor\partial \Omega_{i}=0\right.$ in $\Omega$. Since the value of the right hand side of (2.7) is unchanged even if we restrict $\left\{\Omega_{i}\right\}$ so that $G_{B_{i}}\left\lfloor\partial \Omega_{i}=0\right.$ in $\Omega$, the formula (2.6) now yields (2.7).

The method developed here applies to the modified defect energy

$$
J_{+}^{3}(\nabla u)=\int_{\Sigma}|[\nabla u]|^{3} H\left(\nabla u^{+} \cdot \nu\right) d \mathcal{H}^{1},
$$

where $H$ is the Heaviside function: $H(\tau)=1$ for $\tau>0, H(\tau)=0$ otherwise. The value $H\left(\nabla u^{+} \nu\right)$ is independent of the choice of $\nu$. It only depends on points of $\Sigma$ and behaviour of $u$ near $\Sigma$. This defect energy only measures the jump discontinuities where $\partial u / \partial \nu>0$.

Theorem 2.5 (Representation). Assume that $u \in B G V(\Omega)$ satisfies $|\nabla u|=1$ $\left(\mathcal{L}^{2}\right.$-a.e. $)$ in $\Omega$. Then

$$
\begin{array}{r}
\int_{\Sigma \cap A}|[\nabla u]|^{3} H\left(\nabla u^{+} \cdot \nu\right) d \mathcal{H}^{1}=6 \sup \left\{\sum _ { i = 1 } ^ { m } \int _ { \Omega \cap A } d \left(\left(G_{B_{i}}(\nabla u)\right)_{+}\left\lfloor\Omega_{i}\right) ;\right.\right. \\
\left\{\Omega_{i}\right\}_{i=1}^{m} \in \Lambda, B_{i}=\left\{\xi_{i}, \eta_{i}\right\} \in \mathcal{B}_{+} \text {with the condition }(C): \\
\text { the argument } \left.\sigma \text { of } \nu \text { on } \Omega_{i} \cap \Sigma \text { from } \xi_{i} \text { is less than } \pi / 4\right\}
\end{array}
$$


for a set $A$ in $\Omega$. In particular

$$
\begin{array}{r}
J_{+}^{3}(\nabla u)=6 \sup \left\{\sum _ { i = 1 } ^ { m } \int _ { \Omega } d ( G _ { B _ { + } } ( \nabla u ) ) \left\lfloor\Omega_{i} ;\left\{\Omega_{i}\right\}_{i=1}^{m} \in \Lambda,\right.\right. \\
\left.B_{i}=\left\{\xi_{i}, \eta_{i}\right\} \in \mathcal{B}_{+} \text {satisfying the condition }(C)\right\} .
\end{array}
$$

Both formulas are still valid even if $\Lambda$ is replaced by $\Lambda_{0}$. Here $\mu_{+}$for signed measure $\mu$ denotes the nonnegative part of $\mu$.

Theorem 2.6 (Lower semicontinuity). Assume that $u^{\varepsilon} \in B G V(\Omega)(\varepsilon \geq 0)$ satisfies $\left|\nabla u^{\varepsilon}\right|=1\left(\mathcal{L}^{2}\right.$-a.e.) in $\Omega$. If $\nabla u^{\varepsilon}$ converges to $\nabla u^{0}$ in $L^{1}(\Omega)$ (or $\mathcal{L}^{2}$-a.e.), then

$$
J_{+}^{3}\left(\nabla u^{0}\right) \leq \liminf _{\varepsilon \rightarrow 0} J_{+}^{3}\left(\nabla u^{\varepsilon}\right)
$$

The proof is essentially similar to that of Theorems 2.1 and 2.2. For example we have a representation of $\left(G_{B}\right)_{+}$corresponding to Lemma 2.4. The proof is essentially contained in that of Lemma 2.3.

Lemma 2.7. Let $u \in B G V(\Omega)$ satisfy $|\nabla u|=1\left(\mathcal{L}^{2}\right.$-a.e. $)$ in $\Omega$. Then

$$
\int_{\Sigma \cap A} d\left(G_{B}(\nabla u)\right)_{+}=\frac{4}{3} \int_{\Sigma \cap A}\left(\cos 2 \sigma \cos ^{3} \theta\right)_{+} d \mathcal{H}^{1}, \quad\left(a_{+}=\max (a, 0)\right)
$$

for a subset $A$ of $\Omega$ with $B=\{\xi, \eta\}$. Here $\sigma$ and $\sigma+\theta$ are arguments of $\nu$ and $\nabla_{B} u^{+}$ with respect to the coordinate system associated to $B$, respectively. If $|\sigma|<\pi / 4$, then the right hand side equals

$$
\frac{4}{3} \int_{\Sigma \cap A} \cos 2 \sigma\left(\cos ^{3} \theta\right)+d \mathcal{H}^{1}
$$

If we note Lemma 2.7 and

$$
\int_{\Sigma \cap A}|[\nabla u]|^{3} H\left(\nabla u^{+} \cdot \nu\right) d \mathcal{H}^{1}=8 \int_{\Sigma \cap A}(\cos \theta)_{+}^{3} d \mathcal{H}^{1},
$$

we observe that the rest of the proof of Theorem 2.5 parallels that of Theorem 2.1. Theorem 2.6 follows from Theorem 2.5 in the same way to derive Theorem 2.2 from Theorem 2.1 since we have

$$
\begin{aligned}
\int_{\Omega_{i}} d\left(G_{B}(\nabla u)\right)_{+} & =\sup \left\{\int_{\Omega_{i}} \varphi d G_{B_{i}} ; \varphi \in \Phi_{i}, \varphi \geq 0\right\} \\
& =\sup \left\{\int_{\Omega_{i}}\left(-\nabla_{B_{i}} \varphi \cdot \Xi\left(u_{\xi_{i}}, u_{\eta_{i}}\right)\right) d \mathcal{L}^{2} ; \varphi \in \Phi_{i} ; \varphi \geq 0\right\} .
\end{aligned}
$$


We safely omit the details to the reader.

Remark 2.8 Our lower semicontinuity results (Theorems 2.2 and 2.6) are rather surprising since for $\beta>3$

$$
J_{+}^{\beta}(\nabla u)=\int_{\Sigma}|[\nabla u]|^{\beta} H\left(\nabla u^{+} \cdot \nu\right) d \mathcal{H}^{1}
$$

is not lower semicontinuous with respect to the $L^{1}$ convergence of $\nabla u$ satisfying $|\nabla u|=1$ in $\Omega$. We give a counterexample. Let $\Omega$ be a rectangle of form

$$
\Omega=\{(x, y) ;|y|<1,|x|<2\}
$$

Let $u^{0}$ be the minus of distance function from the boundary $\partial \Omega$, i.e.

$$
u^{0}(x, y)=-\operatorname{dist}((x, y), \partial \Omega)
$$

Evidently, the jump discontinuity $\Sigma$ of $u^{0}$ is of form

$$
\begin{aligned}
& \Sigma=L \cup S_{+} \cup S_{-}, \\
& L=\{(x, y) \in \Omega ;|x|<1, y=0\} \\
& S_{ \pm}=\{(x, y) \in \Omega ;|y|= \pm x-1\}
\end{aligned}
$$

and $\nabla u^{+} \cdot \nu>0\left(\mathcal{H}^{1}\right.$-a.e. $)$ on $\Sigma$. To construct a sequence we set

$$
v(x, y)=-\operatorname{dist}((x, y), \partial D) \quad \text { for } \quad(x, y) \in D
$$

where $D$ is a square of form

$$
D=\{(x, y)|| x|<1,| y \mid<1\}
$$

Our $u^{0}$ is rewritten of the form

$$
u^{0}(x, y)= \begin{cases}v(x-1, y), & x \geq 1 \\ |y|-1, & |x| \leq 1 \\ v(x+1, y), & x \leq-1\end{cases}
$$

For $k=1,2, \cdots$ we set

$$
\begin{aligned}
& u^{k}(x, y)=+v\left(2 k\left(x-\frac{j}{k}\right), 2 k y\right)+\frac{1}{2 k}-1, \\
& \text { for }|| x|-j / k| \leq 1 / 2 k,|y| \leq 1 / 2 k, j=0,1, \cdots k
\end{aligned}
$$


and $u^{k}(x, y)=u^{0}(x, y)$ for other $(x, y)$ 's. By definition both $u^{k}$ and $u^{0}$ solves $|\nabla u|=1\left(\mathcal{L}^{2}\right.$-a.e. $)$ in $\Omega$. Moreover, $\nabla u^{k} \rightarrow \nabla u^{0}\left(\mathcal{L}^{2}\right.$-a.e. $)$ in $\Omega$ since the Lebesgue measure of the set of $(x, y)$ where $u^{k}$ does not agree with $u^{0}$ tends to zero as $k \rightarrow \infty$.

We shall prove that

$$
J_{+}^{\beta}\left(\nabla u^{0}\right)>\liminf _{k \rightarrow \infty} J_{+}^{\beta}\left(\nabla u^{k}\right)
$$

for $\beta>3$. Since $u^{k}=u^{0}$ for $|x| \geq 1$, to show (2.17) it suffices to prove

$$
\int_{L}\left|\left[\nabla u^{0}\right]\right|^{\beta} d \mathcal{H}^{1}>\liminf _{k \rightarrow \infty} \int_{D}\left|\left[\nabla u^{k}\right]\right|^{\beta} H\left(\nabla u^{k} \cdot \nu\right) d\left(\mathcal{H}^{1}\left\lfloor\Sigma_{k}\right)\right.
$$

where $\Sigma_{k}$ denotes the jumps of $\nabla u^{k}$ in $D$. By definition (2.16) of $u^{k}$ the set $\Sigma_{k}$ is of form

$$
\begin{aligned}
& \Sigma_{k}=\Sigma_{k}^{-} \cup \Sigma_{k}^{+} \\
& \Sigma_{k}^{-}=\{(x, y), \quad|| x|-j / k|=1 / 2 k,|x|<1,|y| \leq 1 / 2 k \text { for some } j=0,1, \cdots k\} \\
& \Sigma_{k}^{+}=\{(x, y) ;|y|=|| x|-j / k|,|x| \leq 1,|y| \leq 1 / 2 k \text { for some } j=0,1, \cdots k\}
\end{aligned}
$$

On $\Sigma_{k}^{-}$it is easy to see that $\nabla u^{+} \cdot \nu<0$ while $\nabla u^{+} \cdot \nu>0$ on $\Sigma_{k}^{+}$. Thus

$$
\int_{\Sigma_{k}}\left|\left[\nabla u^{k}\right]\right|^{\beta} H\left(\nabla u^{k}, \nu\right) d \mathcal{H}^{1}=\int_{\Sigma_{k}^{+}}\left|\left[\nabla u^{k}\right]\right|^{\beta} d \mathcal{H}^{1}
$$

Since $\left|\left[\nabla u^{k}\right]\right|=\sqrt{2}$ on $\Sigma_{k}^{+}$we see

$$
\int_{\Sigma_{k}^{+}}\left|\left[\nabla u^{k}\right]\right|^{\beta} d \mathcal{H}^{1}=2^{\beta / 2} \mathcal{H}^{1}\left(\Sigma_{k}^{+}\right)=2^{\beta / 2} \cdot 2 \cdot 2 k \cdot \frac{\sqrt{2}}{2 k} \cdot 2=2 \cdot 2^{(\beta+3) / 2},
$$

while

$$
\int_{L}\left|\left[\nabla u^{0}\right]\right|^{\beta} d \mathcal{H}^{1}=2 \cdot 2^{\beta} .
$$

This shows (2.18) if $\beta>3$. (If $\beta=3$ we do not get (2.18) of course.)

Although this example is not enough to prove that $J^{\beta}$ with $\beta>3$ fails to be lower semicontinuous, we conjecture that $J^{\beta}$ for $\beta>3$ is not lower semicontinuous.

Remark 2.9 We conjecture that (2.5) holds for all $u$ satisfying $|\nabla u|=1\left(\mathcal{L}^{2}\right.$-a.e. $)$ in $\Omega$ if the right hand side of (2.5) is finite. We do not know any charactrization of $\nabla u$ with finite right hand side of (2.5) without assuming that $u \in B G V(\Omega)$. 
3. Estimates of limit energy of the Ginzburg-Landau type energy

Let $\Omega$ be a bounded domain in $\mathbf{R}^{2}$ with Lipschitz boundary. We consider two functionals of Ginzburg-Landau type:

$$
\begin{aligned}
& E_{\varepsilon}(\nabla u)=\frac{1}{2} \int_{\Omega}\left\{\varepsilon^{-1}\left(|\nabla u|^{2}-1\right)^{2}+\varepsilon|\nabla \nabla u|^{2}\right\} d \mathcal{L}^{2} \\
& \hat{E}_{\varepsilon}(\nabla u)=\frac{1}{2} \int_{\Omega}\left\{\varepsilon^{-1}\left(|\nabla u|^{2}-1\right)^{2}+\varepsilon|\Delta u|^{2}\right\} d \mathcal{L}^{2}
\end{aligned}
$$

with small parameter $\varepsilon>0$, where

$$
\Delta u=u_{x x}+u_{y y},|\nabla \nabla u|^{2}=u_{x x}^{2}+u_{x y}^{2}+u_{y x}^{2}+u_{y y}^{2}
$$

To fix the idea we impose

$$
\frac{\partial u}{\partial \mathrm{n}}=-1, u=\text { constant on } \partial \Omega
$$

as a boundary condition, where $\mathrm{n}$ is the unit outer normals of $\partial \Omega$, although our theory applies some other boundary conditions. We consider $E_{\varepsilon}$ and $\hat{E}_{\varepsilon}$ as functionals defined on

$$
X=\left\{\nabla u ; u \in H^{2}(\Omega) \text { satisfying }(3.3)\right\}
$$

where $H^{2}(\Omega)$ is the $L^{2}$ type Sobolev space of order two, i.e. $u \in H^{2}(\Omega)$ is equivalent to say that $u, \nabla u, \nabla \nabla u \in L^{2}(\Omega)$. We next recall notion of $\Gamma$-convergence introduced by De Giorgi (see e.g. [DG2], [DM]). Let $\mathcal{E}_{p}$ be a functional on

$$
Z_{p}=\left\{\nabla u ; \nabla u \in L^{p}(\Omega)\right\}, \quad(1 \leq p<\infty)
$$

with values in $\mathbf{R} \cup\{+\infty\}$. A functional $\mathcal{E}_{p}$ is called the $\Gamma^{-}\left(L^{p}(\Omega)\right)$-limit of $E_{\varepsilon}$ as $\varepsilon \rightarrow 0$ if

$$
\mathcal{E}_{p}(\nabla u)=\inf \left\{\liminf _{\varepsilon \rightarrow 0} E_{\varepsilon}\left(\nabla u^{\varepsilon}\right), \nabla u^{\varepsilon} \rightarrow \nabla u \text { in } L^{p}(\Omega)\right\}
$$

In other words

(i) (existence of approximate functions) for each $\nabla u \in Z_{p}$ there is a sequence $\nabla u^{j} \in X$ satifying $\nabla u^{j} \rightarrow \nabla u$ in $L^{p}(\Omega)$ and $\lim _{j \rightarrow \infty} E_{\varepsilon_{j}}\left(\nabla u^{j}\right)=\mathcal{E}_{p}(\nabla u)$ for some subsequence $\left\{\varepsilon_{j}\right\}_{j=1}^{\infty}$ converging to zero;

(ii) (lower semicontinuity) if $\nabla u^{j} \rightarrow \nabla u \in Z_{p}$ with $\nabla u^{j} \in X$, then

$$
\mathcal{E}_{p}(\nabla u) \leq \liminf _{j \rightarrow \infty} E_{\varepsilon_{j}}\left(\nabla u^{j}\right)
$$


for any subsequence $\left\{\varepsilon_{j}\right\}$ converging to zero.

Let $\mathcal{E}_{p}$ and $\hat{\mathcal{E}}_{p}$ be the $\Gamma^{-}\left(L^{p}(\Omega)\right)$-limit of $E_{\varepsilon}$ and $\hat{E}_{\varepsilon}$ as $\varepsilon \rightarrow 0$, respectively. Since $\Omega$ is bounded, by definition $\mathcal{E}_{p} \leq \mathcal{E}_{q}$ for $p \leq q$.

Our goal is to give sharp estimates of $\mathcal{E}_{p}$ and $\hat{\mathcal{E}}_{p}$ from below.

Theorem 3.1. For the $\Gamma$-limit $\mathcal{E}_{p}=\hat{\mathcal{E}}_{p}(1 \leq p<\infty)$ as functionals. If $\mathcal{E}_{p}(\nabla u)<$ $\infty$, then $|\nabla u|=1\left(\mathcal{L}^{2}\right.$-a.e. $)$ in $\Omega$.

Let $\mathcal{F}$ be the functional on $Z_{p}$ of form

$$
\mathcal{F}(\nabla u)=\left\{\begin{aligned}
\sup \left\{\sum_{i=1}^{m} \int_{\Omega} d \mid G_{B_{i}}(\nabla u)\left\lfloor\Omega_{i} \mid ;\right.\right. & \left.\left\{\Omega_{i}\right\} \in \Lambda, B_{i} \in \mathcal{B}_{+}\right\} \\
& \text {if }|\nabla u|=1\left(\mathcal{L}^{2} \text {-a.e. }\right) \text { on } \Omega \text { and } \\
& u=\text { constant on } \partial \Omega, \\
& +\infty \text { otherwise. }
\end{aligned}\right.
$$

We conjecture that $\mathcal{F}=\mathcal{E}_{p}$. Unfortunately, we are unable to prove this identity. We give estimates for $\mathcal{E}_{\boldsymbol{p}}$.

Theorem 3.2. $\mathcal{F} \leq \mathcal{E}_{p}$ on $Z_{p}$ for $p \geq 3$. If $u \in B G V(\Omega)$, then $J^{3}(\nabla u) / 6=$ $\mathcal{F}(\nabla u) \leq \mathcal{E}_{p}(\nabla u)$ for $\nabla u \in Z_{p}(p \geq 3)$.

Remark 3.3 (i) The restriction $p \geq 3$ seems to be technical. For example if the boundedness of $E_{\varepsilon}\left(\nabla u^{\varepsilon}\right)$ implies compactness of $\left\{\nabla u^{\varepsilon}\right\}$ in $L^{q}(\Omega)$ for all $1 \leq q<\infty$, $\nabla u^{\varepsilon} \rightarrow \nabla u$ in $L^{1}(\Omega)$ implies $\nabla u^{\varepsilon} \rightarrow \nabla u$ in $L^{q}(\Omega)$. If so, $\mathcal{E}_{p}$ is independent of $p$. In this paper we do not pursue the problem of compactness.

(ii) In many examples of $\nabla u$ we observe that $\mathcal{F}(\nabla u)=\mathcal{E}_{p}(\nabla u)$ for $p \geq 3$ if $\nabla u$ is regular away from $\Sigma$ so that we can construct approximate functions, although we do not show explicitly in the present paper. This shows that our estimate is sharp. We moreover conjecture that $\mathcal{F}=\mathcal{E}_{p}$ as functionals of $Z_{p}$.

In the rest of this section we shall prove Theorems 3.1 and 3.2. We begin with an integral identity.

Lemma 3.4. For $\varphi \in C_{0}^{1}(\Omega)$ and $u \in H^{2}(\Omega)$ we have

$$
\begin{aligned}
& \int_{\Omega} \varphi u_{x x} u_{y y} d \mathcal{L}^{2}-\int_{\Omega} \varphi u_{x y} u_{x y} d \mathcal{L}^{2} \\
= & -\frac{1}{2} \int_{\Omega} \nabla^{\perp} u \cdot\left\{\left(\nabla^{\perp} \varphi \cdot \nabla\right) \nabla u\right\} d \mathcal{L}^{2},
\end{aligned}
$$


where $\nabla^{\perp} \varphi=\left(\varphi_{y},-\varphi_{x}\right)$.

Proof. Integrating by parts twice yields

$$
\begin{aligned}
\int \varphi u_{x x} u_{y y} & =\int \varphi_{y} u_{x x} u_{y}-\int \varphi u_{x x y} u_{y} \\
& =\int\left(\varphi_{x} u_{y} u_{x y}-\varphi_{y} u_{y} u_{x x}\right)+\int \varphi u_{x y}^{2}
\end{aligned}
$$

where we suppress the domain of integation as well as $d \mathcal{L}^{2}$. Changing the role of $x$ and $y$ in (3.5) we have

$$
\int \varphi u_{x x} u_{y y}=\int\left(\varphi_{y} u_{x} u_{x y}-\varphi_{x} u_{x} u_{y y}\right)+\int \varphi u_{x y}^{2} .
$$

Averaging these two identities yields

$$
\begin{aligned}
\int \varphi u_{x x} u_{y y} & =\frac{1}{2} \int\left\{u_{y}\left(\varphi_{y}\left(u_{x}\right)_{x}-\varphi_{x}\left(u_{x}\right)_{y}\right)-u_{x}\left(\varphi_{y}\left(u_{y}\right)_{x}-\varphi_{x}\left(u_{y}\right)_{y}\right)\right\} \\
& +\int \varphi u_{x y}^{2} \\
& =-\frac{1}{2} \int \nabla^{\perp} u \cdot\left\{\left(\nabla^{\perp} \varphi \cdot \nabla\right) \nabla u\right\}+\int \varphi u_{x y}^{2} \quad \square
\end{aligned}
$$

We approximate the characteristic function $\chi_{\Omega}$ of $\Omega$ by $\varphi \in C_{0}^{1}(\Omega)$ in Lemma 3.4 to get:

Lemma 3.5. For $u \in H^{2}(\Omega)$ we have

$$
\begin{aligned}
& \int_{\Omega} u_{x x} u_{y y} d \mathcal{L}^{2}-\int_{\Omega} u_{x y} u_{x y} d \mathcal{L}^{2} \\
= & \frac{1}{2} \int_{\partial \Omega} \nabla^{\perp} u \cdot\left(\left(n_{y} \partial_{x}-n_{x} \partial_{y}\right) \nabla u\right) d \mathcal{H}^{I}
\end{aligned}
$$

where $\mathbf{n}=\left(n_{x}, n_{y}\right)$ is the unit outward normal of $\partial \Omega$.

Proof of Theorem 3.1. Applying Lemma 3.5 we see

$$
\begin{aligned}
& 2\left(\hat{E}_{\varepsilon}(\nabla u)-E_{\varepsilon}(\nabla u)\right)=2 \varepsilon\left\{\int_{\Omega} u_{x x} u_{y y} d \mathcal{L}^{2}-\int_{\Omega} u_{x y} u_{x y} d \mathcal{L}^{2}\right\} \\
= & \varepsilon \int_{\partial \Omega} \nabla^{\perp} u \cdot\left(\left(n_{y} \partial_{x}-n_{x} \partial_{y}\right) \nabla u\right\} d \mathcal{H}^{1} .
\end{aligned}
$$

Since $u$ satisfies the boundary condition (3.3),

$$
\nabla u=-\mathbf{n}
$$


so that $\nabla^{\perp} u=\left(-n_{y}, n_{x}\right)$ is a unit tangent vector $\tau$ of $\partial \Omega$. Since $\partial_{\tau} \mathbf{n}=k \tau$ where $k$ is the curvature in the direction of $-\mathbf{n}$, we see

$$
2\left(\hat{E}_{\varepsilon}(\nabla u)-E_{\varepsilon}(\nabla u)\right)=\varepsilon \int_{\partial \Omega} \tau \cdot \partial_{\tau} \mathbf{n} d \mathcal{H}^{1}=\varepsilon \int_{\partial \Omega} k d \mathcal{H}^{1}
$$

Since $\int k d \mathcal{H}^{1}$ is independent of $u \in X$, this implies $\mathcal{E}_{p}=\hat{\mathcal{E}}_{p}$.

The assertion that $\mathcal{E}_{P}(\nabla u)<\infty$ implies $|\nabla u|=1$ in $\Omega$ is standard. We give its proof for completeness. Suppose that $\mathcal{E}_{p}(\nabla u)<\infty$. Then part (i) of the definition of $\mathcal{E}_{p}$ guarantees that there is a sequence $\nabla u^{j} \rightarrow \nabla u$ in $L^{p}(\Omega)$ that satisfies

$$
\lim _{j \rightarrow \infty} E_{\varepsilon_{j}}(\nabla u)=\mathcal{E}_{p}(\nabla u)
$$

for some subsequence $\left\{\varepsilon_{j}\right\}_{j=1}^{\infty}$ converging to zero. Since $E_{\varepsilon_{j}}\left(\nabla u^{j}\right)$ is bounded,

$$
\int_{\Omega} \frac{\left(\left|\nabla u^{j}\right|^{2}-1\right)^{2}}{\varepsilon_{j}} d \mathcal{L}^{2}
$$

is bounded as $j \rightarrow \infty$. Since $\varepsilon_{j} \rightarrow 0$ as $j \rightarrow \infty$, this implies $\left|\nabla u^{j}\right|^{2} \rightarrow 1$ in $L^{2}(\Omega)$, Taking a subsequence if necessary, we may conclude that $\nabla u^{j} \rightarrow \nabla u\left(\mathcal{L}^{2}\right.$-a.e. $)$ and $\left|\nabla u^{j}\right| \rightarrow 1\left(\mathcal{L}^{2}\right.$-a.e. $)$ in $\Omega$. Thus $|\nabla u|=1\left(\mathcal{L}^{2}\right.$-a.e. $)$ in $\Omega$.

Proof of Theorem 3.2. We may assume $p=3$ and $\mathcal{E}_{3}(\nabla u)<\infty$. By Theorem 3.1 we have $|\nabla u|=1$ in $\Omega$. It is not difficult to prove $u=$ const on $\partial \Omega$ since there is $\nabla u^{\varepsilon}$ converging to $\nabla u$ in $L^{3}(\Omega)$ with $\nabla u^{\varepsilon} \in X$. Thus to show $\mathcal{F} \leq \mathcal{E}_{3}$ it suffices to prove

$$
\sum_{i=1}^{m} \int_{\Omega} d \mid G_{B_{i}}(\nabla u)\left\lfloor\Omega_{i} \mid \leq \mathcal{E}_{3}(\nabla u)\right.
$$

with $\left\{\Omega_{i}\right\}_{i=1}^{m} \in \Lambda_{0}, B_{i} \in \mathcal{B}_{+}$. As in the proof of Theorem 2.1 (ii)

$$
\int_{\Omega_{i}} d \mid G_{B_{i}}(\nabla u)\left\lfloor\Omega_{i}\left|=\sup _{\varphi_{i} \in \Phi_{i}}\right| \int_{\Omega_{i}} \nabla_{B_{i}} \varphi_{i} \cdot \Xi\left(u_{\xi_{i}}, u_{\eta_{i}}\right) d \mathcal{L}^{2} \mid, B_{i}=\left\{\xi_{i}, \eta_{i}\right\} .\right.
$$

Since the last term is lower semicontinuous under $L^{3}(\Omega)$ convergence of $\nabla u$, to show (3.6) it suffices to prove

$$
\left|\int_{\Omega_{i}} \nabla_{B_{i}} \varphi_{i} \cdot \Xi\left(u_{\xi_{i}}^{\varepsilon}, u_{\eta_{i}}^{\varepsilon}\right) d \mathcal{L}^{2}\right| \leq E_{\varepsilon}\left(\nabla u^{\varepsilon}, \Omega_{i}\right)+o(1)
$$


for $\nabla u^{\varepsilon} \in X$ converging to $\nabla u$ in $L^{3}(\Omega)$ with bounded $E_{\varepsilon}\left(\nabla u^{\varepsilon}, \Omega_{i}\right)$ as $\varepsilon \rightarrow 0$, where

$$
E_{\varepsilon}\left(\nabla u, \Omega_{i}\right)=\frac{1}{2} \int_{\Omega_{i}}\left\{\varepsilon^{-1}\left(|\nabla u|^{2}-1\right)^{2}+\varepsilon|\nabla \nabla u|^{2}\right\} d \mathcal{L}^{2} .
$$

We shall prove (3.7). We may assume that $B_{i}=I$ by a rotation of coordinates. Integrating by parts and using $(2.10)$ yields

$$
\begin{aligned}
& -\int_{\Omega_{i}} \nabla \varphi \cdot \Xi\left(u_{x}, u_{y}\right) d \mathcal{L}^{2}=\int_{\Omega_{i}} \varphi \operatorname{div}\left(\Xi\left(u_{x}, u_{y}\right)\right) d \mathcal{L}^{2} \\
= & \int_{\Omega_{i}} \varphi\left(1-|\nabla u|^{2}\right)\left(u_{x x}-u_{y y}\right) d \mathcal{L}^{2},
\end{aligned}
$$

where $\varphi=\varphi_{i} \in \Phi$. Since $|\varphi| \leq 1$, the Cauchy inequality yields

$$
\begin{aligned}
\mid \int_{\Omega_{i}} \nabla \varphi & \cdot \Xi\left(u_{x}, u_{y}\right) d \mathcal{L}^{2} \mid \leq \frac{1}{2} \int_{\Omega_{i}}\left\{\varepsilon^{-1}\left(1-|\nabla u|^{2}\right)^{2}+\varepsilon \varphi^{2}\left(u_{x x}-u_{y y}\right)^{2}\right\} d \mathcal{L}^{2} \\
= & \frac{1}{2} \int_{\Omega_{i}}\left\{\varepsilon^{-1}\left(1-|\nabla u|^{2}\right)^{2}+\varepsilon \varphi^{2}\left(u_{x x}^{2}+u_{y y}^{2}-2 u_{x y}^{2}\right)\right\} d \mathcal{L}^{2} \\
& \left.+\varepsilon \int_{\Omega_{i}} \varphi^{2}\left(u_{x y}^{2}-u_{x x} u_{y y}\right)\right\} d \mathcal{L}^{2} \\
\leq & E_{\varepsilon}\left(u, \Omega_{i}\right)+K \\
K= & \left.\varepsilon \int_{\Omega_{i}} \varphi^{2}\left(u_{x y}^{2}-u_{x x} u_{y y}\right)\right\} d \mathcal{L}^{2}
\end{aligned}
$$

We apply Lemma 3.4 to get

$$
K=\frac{\varepsilon}{2} \int_{\Omega_{i}} \nabla^{\perp} u \cdot\left\{\left(\nabla^{\perp} \psi \cdot \nabla\right) \nabla u\right\} d \mathcal{L}^{2} \quad \text { with } \psi=\varphi^{2} .
$$

Applying the Schwarz inequality, we obtain

$$
|K| \leq \frac{1}{2} \sup _{\Omega_{i}}\left|\nabla^{\perp} \psi\right|\left\|\varepsilon^{1 / 2} \nabla^{\perp} u\right\|_{L^{2}}\left\|\varepsilon^{1 / 2}|\nabla \nabla u|\right\|_{L^{2}} .
$$

Assume now that $\nabla u^{\varepsilon}$ be as in (3.7). Since $E_{\varepsilon}\left(\nabla u^{\varepsilon}, \Omega_{i}\right)$ is bounded as $\varepsilon \rightarrow 0$, we observe that $\left\|\varepsilon^{1 / 2}|\nabla \nabla u|\right\|_{L^{2}}$ is bounded as $\varepsilon \rightarrow 0$ and that $\varepsilon^{-1 / 2}\left\|\left|\nabla u^{\varepsilon}\right|^{2}-1\right\|_{L^{2}}$ is bounded so that $\left\|\nabla u^{\varepsilon}\right\|_{L}^{2}$ is bounded. Thus

$$
\left\|\varepsilon^{1 / 2} \nabla^{\perp} u^{\varepsilon}\right\|_{L^{2}}^{2} \rightarrow 0 \text { as } \varepsilon \rightarrow 0 .
$$

so that $K$ with $u=u^{\varepsilon}$ tends to zero as $\varepsilon \rightarrow 0$. The estimate (3.7) now follows from (3.8) with $u=u^{\varepsilon}$. 
The last assertion now follows from Theorem 2.1.

Remark 3.6. The identity of Lemma 3.5 has been essentially used by W. Jin and $\mathrm{R}$. Kohn [JK], where the right hand side vanishes by boundary conditions. This identity together with estimate (3.8) with $\varphi \equiv 1$ has been used in [JK] to estimate the lower bound of $\liminf _{\varepsilon \rightarrow 0} \min _{X} E_{\varepsilon}$.

Remark 3.7. By the Cauchy inequality it is clear that

$$
E_{\varepsilon}(\nabla u) \geq\left.\int_{\Omega}|| \nabla u\right|^{2}-1|| \nabla \nabla u \mid d \mathcal{L}^{2} .
$$

If $\nabla u$ is a scalar-valued function, the Gamma limit of $E_{\varepsilon}$ (with volume constraint on $\left.\int_{\Omega} u d \mathcal{L}^{2}\right)$ equals the right hand side as proved in $[\mathrm{M}]$ and $[\mathrm{St}]$ if it is interpreted in an appropriate way. There is a general way to interpret the right hand side for $u \in B G V(\Omega)$ proposed in [AG3] (see also [AG4]). However, we do not know whether the interpretation of [AG3] agrees with $J^{3} / 6$.

\section{Acknowledgement}

The second author is grateful to Professor Robert Kohn for showing his up-todate results with $\mathrm{W}$. Jin and bringing several interesting articles related to thin film and magnetic walls to his attention as well as valuable comments on this project when the second author visited the Courant Institute in June of 1996. This work was done while the first author visited the Mathematics Department of Hokkaido University during 1996/1997. Its hospitality is gratefully acknowledged, as is support from the Japan Society for the Promotion of Science through longterm invitation fellowship program. The second author was partly supported by the NISSAN SCIENCE FOUNDATION and the Japanese Ministry of Education, Science, Sports and Culture through grant no.08874005, 09242201 for scientific research.

\section{REFERENCES}

[Al] G. Alberti, Rank one property for derivatives of functions with bounded variation, Proc. Roy. Soc. Edinburgh Sect. A. 123 (1993), 239-274.

[AG1] P. Aviles and Y. Giga, A mathematical problem related to the physical theory of liquid crystal configurations, Proc. Centre Math. Anal. Austral. Nat. Univ. 12 (1987), 1-16.

[AG2] P. Aviles and Y. Giga, Singularities and rank one properties of Hessian measures, Duke Math. J. 58 (1989), 441-467. 
[AG3] P. Aviles and Y. Giga, Variational integrals on mappings of bounded variation and their lower semicontinuity, Arch. Rational Mech. Anal. 115 (1991), 201-255.

[AG4] P. Aviles and Y. Giga, Minimal currents, greodesic and relaxation of variational integrals on mappings of bounded variation, Duke Math. J. 67 (1992), 517-538.

[AG5] P. Aviles and Y. Giga, The distance function and defect energy, Proc. Roy. Soc. Edinburgh Sect. A. 126 (1996), 923-938.

[DM] G. Dal Maso, An introduction to $\Gamma$-convergence, Birkhäuser (1993).

[DG1] E. De Giorgi, Nuovi theremi relativi alle misure $(r-1)$-dimensionale inn uno spazio ad $r$ dimension, Ricerche Mat. 4 (1995), 95-113.

[DG2] E. De Giorgi, G-operators and $\Gamma$-convergence, Proceedings of ICM Poland, 1983, Warszawa 2(1983), 1175-1191.

[F] H. Federer, Geometric Measure Theory, Springer (1969).

[Giu] E. Giusti, Minimal surfaces and functions of bounded variations, Boston Birkhauser, 1984.

[JK] W. Jin and R. Kohn, In preparation.

[KM1] R. Kohn and S. Muller, Relaxation and regularization of nonconvex variational problems, Rend. Semi. Mat. Fis. Univ. Milano 62 (1992), 89-113.

[KM2] R. Kohn and S. Muller, Surface energy and microstructure in coherent phase transition, Comm. Pure Appl. Math. 47 (1994), 405-435.

[L] P. L. Lions, Generalized Solutions of Hamilton-Jacobi Equations, Boston, Pitman Advanced Publ. Program, 1982.

[M] L. Modica, The gradient theory of phase transitions and the minimal surface criterion, Arch. Rational Mech. Anal. 98 (1987), 123-142.

[OG] M. Ortiz and G. Gioia, The morphology and folding patterns of bucking driven thin-film filters, J. Mech. Phy. Solids 42 (1994), 531-559.

[SK] J. Sethna and M. Kleman, Spheric domains in smectic liquid crystals, Phys. Rev. A. 26 (1982), 3037-3040.

[S] L. Simon, Lectures on geometric measure theory, Canberra, Proc. Centre Math. Anal. Austral. Nat. Univ. 31983.

[St] P. Sternberg, The effect of a singular perturbation on nonconvex variational problems, Arch. Rational Mech. Anal. 101 (1988), 209-260.

[V] A. I. Vol'pert, The spaces BV and quasilinear equations, Math. USSR. Sbornik 2 (1967), 225-267.

[VB] H. A. M. van den Berg, Self-consistent domain theory in soft-ferromagnetic media II, Basic domain structure in thin film objects, J. Appl. Phys. 60 (1986), 1104-1113.

$\left[\mathrm{VB}^{2}\right]$ H. A. M. van den Berg and A. H. J. van den Brandt, Self-consistent domain theory in soft-ferromagnetic media III, Composite structures in thin-film objects, J. Appl. Phys. 62 (1987), 1952-1959. 